\title{
Evaluating Complexity, Code Churn, and Developer Activity Metrics as Indicators of Software Vulnerabilities
}

\author{
Yonghee SHIN, Student Member, IEEE, Andrew MENEELY, Student Member, IEEE, \\ Laurie WILLIAMS, Member, IEEE, and Jason OSBORNE
}

\begin{abstract}
Post-release detection of a software vulnerability does not only cost a company money to fix, but also results in loss of reputation and damaging litigation. Techniques to prevent and detect vulnerabilities prior to release, therefore, are valuable. We performed empirical case studies on two large, widely-used open source projects: the Mozilla Firefox web browser and the Red Hat Enterprise Linux kernel. We investigated whether software metrics obtained early in the software development life cycle are discriminative of vulnerable code locations, and can guide actions for an organization to take for improvement of code and development team. We also investigated whether the metrics are predictive of vulnerabilities so that prediction models can prioritize validation and verification efforts. The metrics fall into three categories: complexity, code churn, and developer activity metrics. The results indicate that the metrics are discriminative and predictive of vulnerabilities. The predictive model on the three categories of metrics predicted $70.8 \%$ of the known vulnerabilities by selecting only $\mathbf{1 0 . 9 \%}$ of the project's files. Similarly, the model for the Red Hat Enterprise Linux kernel found $68.8 \%$ of the known vulnerabilities by selecting only $13.0 \%$ of the files.
\end{abstract}

Index Terms-- Software Security, Software Vulnerability, Process metrics, Product metrics, Fault Prediction, Vulnerability Prediction.

\section{INTRODUCTION}

A single security problem can cause severe damage to an organization by not only incurring large costs for late fixes, but by losing invaluable assets and credibility and leading to legal issues. Annual world-wide losses caused from cyber attacks have been reported to be as high as \$226 billion [8]. Loss in stock market value in the days after an attack is estimated at \$50 million to \$200 million per organization [8]. Therefore, organizations must prioritize vulnerability detection efforts and prevent vulnerabilities from being injected.

One way of identifying the most vulnerable code locations is to use characteristics of the software product itself. Perhaps complex code is more likely to be vulnerable than simple code.

Manuscript received March 18, 2009. This work was supported in part by National Science Foundation Grant No. 0716176 and the U.S. Army Research Office (ARO) under grant W911NF-08-1-0105 managed by NCSU Secure Open Systems Initiative (SOSI).

Y. Shin is with North Carolina State University, Raleigh, NC 27695 USA (telephone: 919-946-0781, e-mail: yonghee.shin@ ncsu.edu).

A. Meneely is with North Carolina State University, Raleigh, NC 27695 USA (e-mail: apmeneel@ncsu.edu).

L. Williams is with North Carolina State University, Raleigh, NC 27695 USA (e-mail: williams@ @sc.ncsu.edu).

J. Osborne is with North Carolina State University, Raleigh, NC 27695 USA (e-mail: jaosborn@ @stat.ncsu.edu). 
Another way of identifying the most vulnerable code locations is to use characteristics of the process of code development. Perhaps code that has undergone recent changes (i.e. code churn) might have new vulnerabilities. Perhaps files worked on by software developers who work alone are more likely to be vulnerable than the files worked on by collaborating developers. These characteristics of software product and process can be quantified by complexity, code churn, and developer activity metrics $[4,24,25,28]$. Complexity metrics measure the structural complexity of code and design such as the number of branches in code. Code churn metrics measure the number of check-ins and amount of code changes occurred during development of code. Developer activity metrics quantify the relationship between developers and code.

When metrics are discriminative of vulnerabilities (e.g. a high value of a metric indicates a vulnerability), insight can be provided for development teams to take actions to prevent vulnerabilities from being injected by restructuring code or reorganizing the development team. We call discriminative metrics that can be used by organizations to drive improvement actionable discriminative metrics $(A D M)$. If, for example, complex code is likely to be vulnerable, then development teams can use this fact to avoid unnecessarily complex code. Alternatively, code churn is often inevitable and metrics of code churn do not offer clear recommendations to developers to improve software quality. Therefore, we classify code churn metrics as non-actionable.

We call metrics that can be used during development to predict the reliability of a product or the location of vulnerabilities predictive metrics [2]. Predictive metrics can be used to prioritize inspection and testing efforts to economically detect vulnerabilities.

The goal of this paper is twofold: (a) to evaluate whether complexity and developer activity metrics can be demonstrated to be actionable discriminative metrics to prevent software 
vulnerabilities; and (b) to evaluate whether complexity, code churn, and developer activity metrics are predictive of vulnerable locations of code. To complete this goal, we performed empirical studies on two widely-used, large scale open source projects: the Mozilla Firefox ${ }^{1}$ web browser and the Linux kernel $^{2}$ as distributed in Red Hat Enterprise Linux ${ }^{3}$. We analyzed 34 releases of Mozilla Firefox and one release of Red Hat Linux that consist of over three millions lines of source code in each release. We collected the reported vulnerability information spanning four years. From 197 vulnerability reports for Mozilla Firefox we identified 1197 instances of file changes to fix vulnerabilities. From 192 vulnerability reports for Red Hat we identified 258 instances of file changes to fix vulnerabilities. We also collected complexity, code churn, and developer activity metrics from the two projects and performed an empirical evaluation of the metrics.

The rest of the paper is organized as follows: Section II defines background terms used in this paper. Section III provides related work. Section IV discusses the hypotheses behind the metrics and provides our methodology for evaluating the hypotheses. In Sections V and VI, we describe the empirical case studies on the Mozilla Firefox web browser and the Red Hat Enterprise Linux kernel. Section VII discusses our observations from the case studies and Section VIII discusses the threats to validity of our study. Section IX summarizes our work.

\section{BACKGROUND}

This section describes the terms that we will use in this paper.

\section{A. Internal and External Metrics}

Since software quality, such as reliability or security, can be measured only after release, metrics that are available early in the development life cycle can be used to estimate software

\footnotetext{
${ }^{1}$ http://www.mozilla.com/firefox/

${ }^{2} \mathrm{http}: / / \mathrm{kernel}$. org/

${ }^{3}$ http://www.redhat.com/rhel/
} 
quality prior to release. Internal metrics measure the "non-executable software product during its development stages" such as code and design specification [16]. An external metric measures "the quality of the software product by measuring the behavior of the system" [16]. The internal metrics we use in this study are complexity, code churn, and developer activity. We use these internal metrics as predictors of the external metric of discovered software vulnerabilities. The external metric we are evaluating in this study is nominal: whether or not a file is vulnerable or neutral. We consider a file to be vulnerable if the file was found to have at least one vulnerability that required a patch after release. A vulnerability is "an instance of a [fault] in the specification, development, or configuration of software such that its execution can violate an [implicit or explicit] security policy" [19].

\section{B. Discriminative Power and Predictability}

Discriminative power is defined as the ability to "discriminate between high-quality software components and low-quality software components" [2]. Discriminative power is used for quality control of software by evaluating software components against predetermined critical values of metrics and by identifying components that fall outside quality limits [35]. In our study, discriminative power is the ability to discriminate vulnerable code from neutral code.

Predictability is defined as the ability of a metric measured at time $\mathrm{T} 1$ to predict the software quality at time T2 with required accuracy. Whether the required accuracy is satisfied or not is determined by testing whether the predicted quality and the actual quality of software is within a certain error range [2].

Note that while both of discriminative power and predictability evaluate the ability to classify code as vulnerable or neutral, their purposes are different. The main purpose for evaluating discriminative power in this study is to take proactive actions to change the measures of metrics 
to improve software security, for example, by redesigning code based on the measures of metrics or by reorganizing the team. The main purpose for evaluating predictability in this study is to take corrective actions on the vulnerabilities found in the code locations predicted as vulnerable by further inspection or testing. Discriminative power and discriminant analysis, a statistical modeling technique that will be explained in Section IV, are different in our study. While discriminant analysis can be used to evaluate both of discriminative power and predictability, we used discriminant analysis only for the purpose of prediction in our study.

\section{Binary Classification Evaluation Criteria}

We perform binary classification to predict vulnerable files. A binary classifier can make two possible errors: false positives (FP) and false negatives (FN). A FP is the classification of a neutral file as a vulnerable file, and a FN is the classification of a vulnerable file as neutral. A correctly classified vulnerable file is a true positive (TP), and a correctly classified neutral file is a true negative (TN). A FP may cause a team to do additional testing or inspection of a file only to find no vulnerabilities. A FN may allow a vulnerability to escape to the field after release. For evaluating binary classification models, we use recall, precision, inspection rate, and vulnerability rate.

Recall $(\mathrm{R})$ is defined as the percentage of vulnerable files found: $R=T P^{*} 100 /(T P+F N)$.

Precision $(\mathrm{P})$ is defined as the percentage of correctly predicted vulnerable files: $P=T P^{*} 100 /(T P+F P)$.

Inspection rate (IR) is the percentage of files that were classified as vulnerable:

$I R=(T P+F P)^{*} 100 /(T P+T N+F P+F N)$. 
The inspection rate represents the percentage of files required for inspection or testing to achieve the reported recall. For example, a $\mathrm{IR}=20 \%$ and $\mathrm{R}=80 \%$ means that $20 \%$ of files were classified as vulnerable and those predicted vulnerable files contained $80 \%$ of actual vulnerabilities.

Vulnerability rate (VR) is the percentage of the number of vulnerabilities that were included in the files classified as vulnerable:

$V R=($ Number of vulnerabilities in the files classified as vulnerable $) * 100 /($ Total number of vulnerabilities).

Note that VR is similar to recall, but differs where recall counts the number of vulnerable files, VR counts the number of vulnerabilities in each file that was classified as vulnerable.

\section{Network Analysis}

In this paper, we use several terms from network analysis [6] and provide their meaning with respect to developer and contribution networks in Section IV. Network analysis is the study of characterizing and quantifying network structures, represented by graphs [6]. In network analysis, vertices of a graph are called nodes, and edges are called connections. A sequence of non-repeating, adjacent nodes is a path, and a shortest path between two nodes is called a geodesic path (note that geodesic paths are not necessarily unique).

Centrality metrics are used to quantify the location of one node relative to other nodes in the network. The centrality metrics we use for developer activity are degree, closeness, and betweenness metrics. The degree metric is defined as the number of neighbors directly connected to a node. The closeness centrality of node $v$ is defined as the average distance from $v$ to any other node in the network that can be reached from $v$. The betweenness centrality [6] of node $v$ is defined as the number of geodesic paths that include $v$. 
EDIT) $<$

Cluster metrics are used to measure the strength of interconnection between groups of nodes. A cluster of nodes is a set of nodes such that there are more edges within a set of nodes (intra-set edges) than edges between a set and other sets of nodes (inter-set edges). The cluster metric we use for developer activity is edge betweenness [13]. The edge betweenness of edge $e$ is defined as the number of geodesic paths which pass through $e$. Since clusters have many intra-cluster edges, edges within clusters have a low betweenness; conversely, edges between two clusters have a high betweenness [13].

\section{RELATED WORK}

This section introduces a few studies on software vulnerability prediction. Then we provide some prior studies that have used complexity, code churn, and developer metrics to predict faults.

\section{A. Vulnerability Prediction}

Alhazmi et al. [3] investigated whether the number of latent vulnerabilities in a software system can be predicted. The researchers analyzed the Windows and Red Hat Enterprise Linux operating systems and observed that the ratio of vulnerabilities to the total number of faults falls in the range of $1 \% \sim 5 \%$. If this ratio is constantly true across projects, the discovered vulnerability densities can be a useful indicator to estimate the efforts to find undiscovered vulnerabilities. While their study is useful for estimating the effort required to identify and correct undiscovered security vulnerabilities, our approach provides specific locations of the vulnerabilities in source code.

Neuhaus et al. [31] introduces a tool, Vulture, to automatically map vulnerability reports to vulnerable components in the Mozilla open source code. Using the tool, they observed common 
patterns of imports (\#include) and function calls in vulnerable components using pattern mining techniques. Vulture identified $45 \%$ of all vulnerable components (recall) using import analysis, and $70 \%$ of all identified vulnerable components were true vulnerable components (precision) using function call analysis. Vulture was also able to identify $82 \%$ of actual vulnerabilities in the top $30 \%$ of files ranked in the order of predicted vulnerabilities. Our study was also performed on Mozilla, but using different metrics that can be obtained from development process as well as from source code.

Gegick et al. [12] modeled vulnerabilities in a large commercial telecommunications software system. These researchers used source lines of code, alert density from a statistic analysis tool, and code churn information. One of the models they tried identified $100 \%$ of vulnerable components with an $8 \%$ false positive rate. However, the model predicted vulnerabilities only at the component level and cannot direct developers to more specific vulnerable code locations. Gegick et al. [11] also investigated whether non-security failure information can be used to predict security failures. In their case study on a Cisco software system, the number of failure reports per component was used as an internal metric to classify vulnerable and neutral components. Their classification and regression tree model was able to identify $57 \%$ of vulnerable components from the top $9 \%$ of components ranked in the order of predicted probability of vulnerability with a $48 \%$ false positive rate. Our study provides predictions at the file level and uses metrics that are available prior to release.

Shin and Williams [36] investigated whether the traditional code level complexity metrics such as cyclomatic complexity can be used as indicators of vulnerabilities in the Mozilla JavaScript Engine. Their results show that the correlations between complexity metrics and vulnerabilities are weak (Spearman $\mathrm{r}=0.30$ at best) but statistically significant. Therefore, 
traditional code level complexity metrics can be used as indicators of vulnerabilities, but further investigation is required with new complexity metrics and other types of metrics including process information.

\section{B. Fault Prediction with complexity, code churn, and developer metrics}

Basili et al. [4] showed the usefulness of object oriented (OO) design metrics to predict faultproneness in a study performed on eight medium-sized information management systems. The logistic regression model with OO design metrics detected $88 \%$ of faulty classes and correctly predicted $60 \%$ of classes as faulty. Briand et al. [7] also used OO design metrics to predict defects and their logistic regression model classified fault-prone classes at over $80 \%$ of precision and found over $90 \%$ of faulty classes. Nagappan et al. [29] found that sets of complexity metrics are correlated with post-release defects using five major Microsoft product components, including Internet Explorer 6. Menzies et al. [25] explored three data mining modeling techniques, OneR, J48, and naïve Bayes, using code metrics to predict defects in MDP, a repository for NASA data set. Their model using naïve Bayes was able to predict defects with $71 \%$ recall and $25 \%$ false positive rate.

Nagappan and Ball [26] investigated the usefulness of code churn information on Windows Server 2003 to estimate post-release failures. The Pearson correlation and Spearman rank correlation between estimated failures and actual post-release failures were 0.889 and 0.929 respectively for the best model. As an effort to improve the performance of prediction, several studies have been performed with combined models of complexity, code churn, and other metrics to improve the accuracy of prediction. Ostrand et al. [33] used code churn information together with other metrics including lines of source code, file age, file type, and prior fault history. They found that $83 \%$ of faults were in the top $20 \%$ of files ranked in the order of 
predicted faults using negative binomial regression. Nagappan et al. [27, 28] also performed empirical case studies on fault prediction with Windows XP and Windows Server 2003 using code churn metrics and code dependency within and between modules. Both studies used a multiple linear regression model on principal components and the Spearman rank correlations between actual post-release failures and estimated failures were 0.64 and 0.68 at the best cases, respectively.

We use two concepts to measure developer activity: developer networks and contribution networks. The concept of a developer network has come from several sources, including [14, 24]. Gonzales-Barahona and Lopez-Fernandez [14] were the first to propose the idea of creating developer networks as models of collaboration from source repositories to differentiate and characterize projects. Meneely et al. [24] applied social network analysis to the developer network in a telecommunications product to predict failures in files. Pinzger et al. [34] were the first to propose the contribution network as a quantification of the direct and indirect contribution of developers on specific resources of the project. Pinzger et al. found that files that were contributed to by many developers, especially by developers who were making many different contributions themselves, were found to be more failure-prone than files developed in relative isolation. Other efforts exist $[15,30,41]$ to quantify developer activity in projects, mostly via counting the number of distinct developers who changed a file as we did in our study.

\section{Methodology}

This section explains our methodology to explore the relationship between complexity, code churn, and developer activity metrics and software vulnerabilities. For this purpose, we examine the discriminative power and predictability of these metrics, as defined in Section II. First we 
provide the hypotheses for discriminative power and predictability of the metrics. Then, we provide the evaluation criteria for the hypotheses and also explain our prediction models.

We organize our hypotheses in a three level hierarchy. The top level hypotheses correspond to the three categories of metrics. The second level hypotheses correspond to the smaller subcategories of metrics. The third level hypotheses correspond to discriminative power and predictability for each individual metric. Fig. 1 shows the first and second level hypotheses. The suffixes “_D” and “_P” represent the hypotheses for discriminative power and for predictability, respectively.

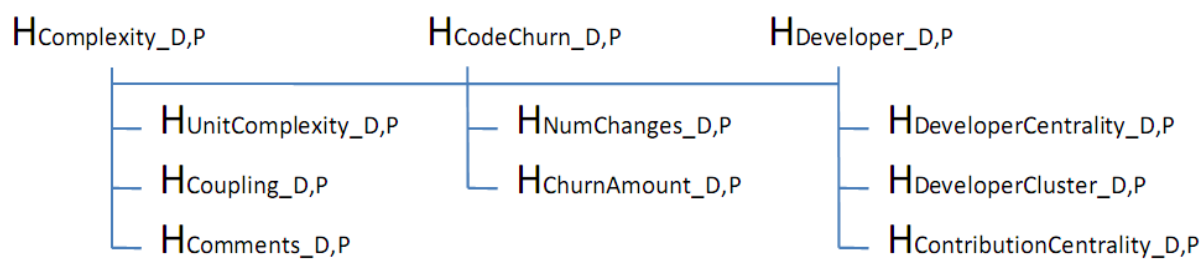

Fig. 1. Hierarchy of hypotheses

\section{A. Hypotheses for Discriminative Power}

\section{1) Complexity Metrics}

Security experts often relate complexity and security $[10,23]$. Complex code is more difficult to understand and test, so developers may have a higher chance of introducing undetected errors. We hypothesize that complexity metrics can indicate security vulnerabilities as follows:

\section{$\boldsymbol{H}_{\text {Complexity_D: }}$ Vulnerable files are more complex than neutral files.}

We break this general hypothesis down into three more specific hypotheses: unit complexity, coupling (interaction complexity), and comment metrics.

Developers might introduce more errors when code is complex and difficult to understand. One characteristic of complex code is having a high number of decisions/branches. Code with many branches would require a higher number of tests for full coverage, which could lead to 
vulnerabilities not being found before release. A popular formulation of this kind of complexity is cyclomatic complexity [22]. For the code with same level of complexity, longer code might be more difficult to understand and maintain than shorter code. Our specific hypothesis related to unit complexity is stated as:

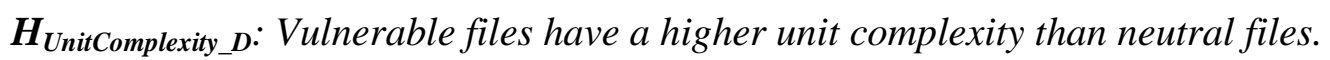

The metrics we use to evaluate $\mathrm{H}_{\text {UnitComplexity_D }}$ and their definitions are provided in Table I.

A second type of complexity is calculated based upon dependencies from one code unit to other code units. If a code unit has many dependencies (or many other units depend on one code unit), that unit is said to be highly coupled. When a unit is highly coupled with other code units that are not closely related in their functions, change in a unit can be unnecessarily propagated to other code units and improve a chance to introduce errors. Our specific hypothesis related to coupling is, therefore, stated as:

$\boldsymbol{H}_{\text {Coupling_D: }}$ Vulnerable files have a higher coupling than neutral files.

The metrics we use to evaluate $\mathrm{H}_{\text {Coupling_D }}$ and their definitions are provided in Table $\mathrm{I}$.

One way to reduce psychological burden imposed by complex code is to add comments [38, 40]. Comments can be used to record the rationale of design decisions so that the rationale is understood when the code is modified by the same developer a long time after the code is implemented or by other developers. Communication by comments between developers is valuable in open source projects for which many developers can contribute on the same code segment. Our specific hypothesis related to comments is therefore stated as:

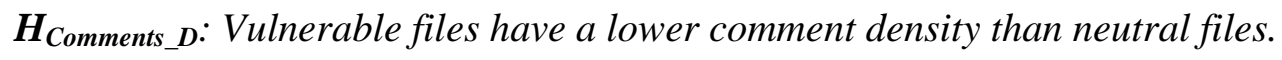


TABLE I

DEFINITIONS OF COMPLEXITY METRICS

\begin{tabular}{|c|c|c|}
\hline $\begin{array}{c}\text { Related } \\
\text { Hypothesis }\end{array}$ & Metric & Definition \\
\hline \multirow{10}{*}{$\mathrm{H}_{\text {UnitComplexity }}$} & CountLineCode & The number of lines of code in a file \\
\hline & CountDeclClass & The number of classes defined in a file \\
\hline & CountDeclFunction & The number of functions defined in a file \\
\hline & CountLineCodeDecl & The number of lines of code in a file devoted to declarations \\
\hline & CountLinePreprocessor & The number of lines of code in a file devoted to preprocessing \\
\hline & SumEssential & $\begin{array}{l}\text { The sum of essential complexity, where essential complexity is } \\
\text { defined as the number of branches after reducing all the programming } \\
\text { primitives such as a for loop in a function's control flow graph into } \\
\text { a node iteratively until the graph cannot be reduced any further. } \\
\text { Completely well-structured code has essential complexity } 1[22,39]\end{array}$ \\
\hline & SumCyclomaticStrict & $\begin{array}{l}\text { The sum of the strict cyclomatic complexity, where strict cyclomatic } \\
\text { complexity is defined as the number of conditional statements in a } \\
\text { function }\end{array}$ \\
\hline & SumMaxNesting & $\begin{array}{l}\text { The sum of the MaxNesting in a file, where MaxNesting is defined as } \\
\text { the maximum nesting level of control constructs such as if or } \\
\text { while statements in a function }\end{array}$ \\
\hline & MaxCyclomaticStrict & The maximum of strict cyclomatic complexity in a file \\
\hline & MaxMaxNesting & The maximum of MaxNesting in a file \\
\hline \multirow{4}{*}{$\mathrm{H}_{\text {Coupling }}$} & SumFanIn & $\begin{array}{l}\text { The sum of FanIn, where FanIn is defined as the number of inputs to } \\
\text { a function such as parameters and global variables }\end{array}$ \\
\hline & SumFanOut & $\begin{array}{l}\text { The sum of FanOut, where FanOut is defined as the number of } \\
\text { assignment to the parameters to call a function or global variables }\end{array}$ \\
\hline & MaxFanIn & The maximum of FanIn \\
\hline & MaxFanOut & The maximum of FanOut \\
\hline $\mathrm{H}_{\text {Comments }}$ & CommentDensity & The ratio of lines of comments to lines of code \\
\hline
\end{tabular}

\section{2) Code Churn}

Code is constantly evolving throughout the development process. Each new change in the system brings a new risk of introducing a vulnerability.

Our first level hypothesis on the relationship between code churn and vulnerabilities is as follows:

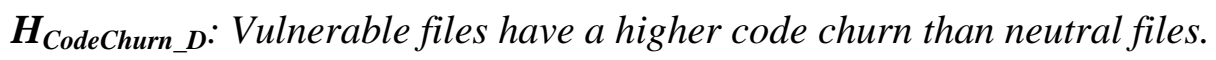

Code churn can be counted in terms of the number of check-ins into a version control system and the number of lines that have been added or deleted by code change. Thus, our second level hypotheses related to code churn metrics are stated as:

$\boldsymbol{H}_{\text {NumChanges_D: }}$ Vulnerable files have more frequent check-ins than neutral files. 


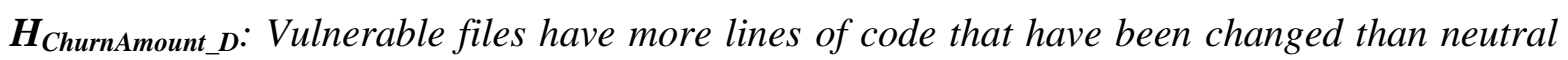
files.

The metrics we use to evaluate $\mathrm{H}_{\text {CodeChurn_D }}$ and their definitions are provided in Table II.

TABLE II

DEFINITIONS OF CODE CHURN METRICS

\begin{tabular}{|l|l|l|}
\hline \multicolumn{1}{c|}{$\begin{array}{c}\text { Related } \\
\text { Hypothesis }\end{array}$} & \multicolumn{1}{c|}{ Metric } & \multicolumn{1}{c|}{ Definition } \\
\hline $\mathrm{H}_{\text {NumChanges }}$ & NumChanges & The number of check-ins for a file since the creation of a file \\
\hline \multirow{2}{*}{$\mathrm{H}_{\text {ChurnAmount }}$} & LinesChanged & The cumulated number of code lines changed since the creation of a file \\
\cline { 2 - 3 } & LinesNew & The cumulated number of new code lines since the creation of a file \\
\hline
\end{tabular}

\section{3) Developer Activity}

Software development is generally performed by development teams working together on a common project. Lack of team cohesion, miscommunications, or misguided effort can all result in problematic software, including security problems [23]. Version control data can be used to construct a developer network and a contribution network based upon "which developer worked on which file," using network analysis defined in Section II. Then we can compute developer centrality, developer clusters, and contribution centrality from those networks.

\section{1) Developer Network Centrality}

In our developer network, two developers are connected if they have both made a change to at least one file in common during the period of time under study. The result is an undirected, unweighted, and simple graph where each node represents a developer and edges are based on whether or not they have worked on the same file during the same release.

A central developer has a high degree, a high betweenness, and/or a low closeness. Refer to [24] for a more in-depth example of how centrality metrics are derived from developer networks. In this study, we are using developer network centrality metrics to evaluate the following hypotheses: 


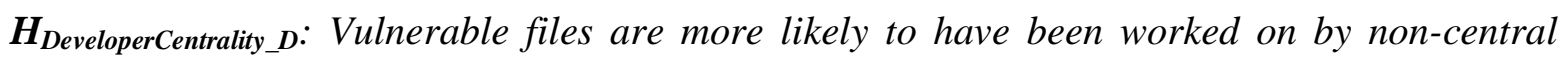
developers than neutral files.

We explain the meaning of high and low values of the metrics that might be associated with high vulnerabilities in Table III. Note that we chose to not study DNMaxDegree, DNMinCloseness, and DNMaxBetweenness because, for example, a high DNMaxDegree means at least one central developer worked on the file, which is not as helpful as knowing that high DNMinDegree denotes that all developers who worked on a file were central. In other words, since most files in a project are worked on by at least one central developer, metrics that indicate that at least one developer worked on a file tend to be homogenous and not particularly useful.

TABLE III

MEANING OF DEVELOPER ACTIVITY METRICS

\begin{tabular}{|c|c|c|c|}
\hline Related Hypothesis & Metric & $\begin{array}{c}\text { Problematic } \\
\text { When }\end{array}$ & Meaning \\
\hline \multirow{6}{*}{$\mathrm{H}_{\text {DeveloperCentrality }}$} & DNMinDegree & Low & \multirow{6}{*}{$\begin{array}{l}\text { File was changed by developers who are } \\
\text { not central to the network }\end{array}$} \\
\hline & DNAvgDegree & Low & \\
\hline & DNMaxCloseness & High & \\
\hline & DNAvgCloseness & High & \\
\hline & DNMinBetweenness & Low & \\
\hline & DNAvgBetweenness & Low & \\
\hline \multirow[b]{2}{*}{$\mathrm{H}_{\text {DeveloperClustering }}$} & DNMaxEdgeBetweenness & High & \multirow{2}{*}{$\begin{array}{l}\text { File was contributed to by more than one } \\
\text { cluster of developers, with few other } \\
\text { files being worked on by each cluster }\end{array}$} \\
\hline & DNAvgEdgeBetweenness & High & \\
\hline \multirow{3}{*}{$\mathrm{H}_{\text {ContributionCentrality }}$} & NumDevs & High & File was changed by many developers \\
\hline & CNCloseness & High & \multirow{2}{*}{$\begin{array}{l}\text { File was changed by developers who } \\
\text { focused on many other files }\end{array}$} \\
\hline & CNBetweenness & High & \\
\hline
\end{tabular}

\section{2) Developer Network Cluster}

Metrics of developer centrality give us information about individual developers, but we also wonder the relationship between groups of developers. In our developer network, a file that is between two clusters was worked on by two groups of developers, and those two groups did not work on many other files in common. By definition, two developer clusters cannot work on 
many files in common, or they would be part of the same cluster. In this study, we are using developer network cluster metrics to evaluate the following hypothesis:

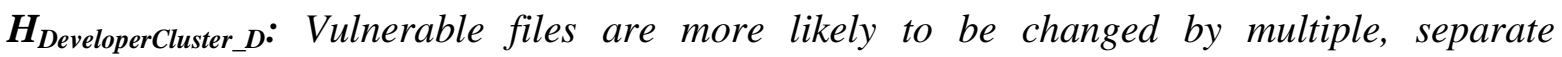
developer clusters than neutral files.

For this study, we use the edge betweenness clustering technique [6] for discovering developer clusters. We are interested in identifying the files that lie between clusters. Since edges and files have a many-to-many relationship, we use the average and maximum of edge betweenness on the developer network as provided in Table III.

\section{3) Contribution Network}

A contribution network is a quantification of the focus made on the relationship between a file and developers instead of relationship between developers. Originally described by Pinzger et al [34], the contribution network uses an undirected, weighted, and bipartite graph with two types of nodes: developers and files. An edge exists where a developer made changes to a file. The weight of an edge is the number of version control commits a developer made to the file. If a file has high centrality, then that file was changed by many developers who made changes to many other files - referred to as an "unfocused contribution." [34] We evaluate the following hypotheses:

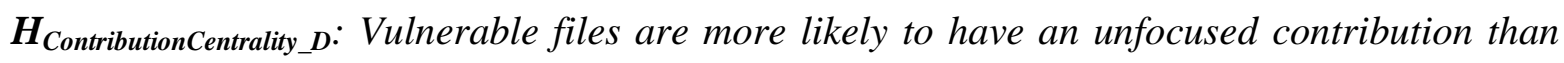
neutral files.

Table III provides the meaning of the contribution network metrics.

\section{B. Hypotheses for Predictability}

When the individual metrics have discriminative power, the individual metrics and the combined set of those individual metrics might be able to predict vulnerabilities with reasonably 
high prediction performance. We hypothesize that complexity, code churn, and developer activity metrics individually and combined together in a model can predict vulnerabilities with over $70 \%$ recall or VR and less than $30 \%$ IR. A model that satisfies these criteria indicates that over $70 \%$ of vulnerable files or vulnerabilities could be detected by inspecting only $30 \%$ of the files. Note that even when precision is very low, the prediction model is helpful for the organization to economically detect vulnerabilities if organization can find high percentage of vulnerabilities with small amount of inspection efforts. Therefore, we considered recall, VR, and IR as the most important evaluation criteria in our study. Since recall and IR often trade off, obtaining high recall and low IR at the same time is a challenging goal. Considering that the economic effect that a single security vulnerability can bring to an organization, we favored high recall and VR to a low IR. However, organizations may choose their own criteria depending on their quality goal for their software products.

\section{Evaluation of Hypotheses}

To evaluate discriminative power of individual metrics, we used Mann-Whitney-Wilcoxon (MWW) test to investigate the null hypothesis that the distribution of a given individual metric measurement among all vulnerable files is the same as that for the metric among all neutral files. The MWW is a non-parametric procedure that enables testing for equality of distributions without making any assumptions about the distributions themselves, like the assumption of normality [20]. To see the association direction (i.e. positively or negatively correlated), we compared the means of the measures of internal metrics for the vulnerable and neutral files. We considered our hypotheses for discriminative power are supported when the results from MWW test are statistically significant at 0.05 level and when the association is in the direction prescribed in the hypotheses. When at least one of the lower level hypotheses is supported, we 
considered the higher level hypothesis is also supported. For example, if $\mathrm{H}_{\text {UnitComplexity_D, }}$ $\mathrm{H}_{\text {Couplng_D}}$, or $\mathrm{H}_{\text {Comments_D }}$ is supported, we considier $\mathrm{H}_{\text {Complexity_D }}$ supported.

To evaluate predictability, we built prediction models using discriminant analysis and Bayesian network modeling. First, we evaluated the predictability for univariate models with individual metrics. Then, we evaluated predictability of a multivariate model by combining metrics into a single model. Because our goal is to predict future vulnerabilities that have not been observed yet, we used cross-validation. In cross-validation, we build or "fit" models using subsets of the data called training data, and evaluate these fitted models on separate data sets, called test data. We performed next-release validation for Mozilla Firefox that has multiple releases. Next-release validation at release $\mathrm{N}$ uses all of the data sets previous to $\mathrm{N}$ as the training data and release $\mathrm{N}$ as the test data. We report the average of the results across all releases. Alternatively, we used ten iterations of three-fold cross validation for Red Hat because we analyzed a single release. Three-fold cross validation validates a model by dividing the data set into thirds, using two thirds as the training data, and one third as the test data. The prediction is then repeated three times with each of the three data sets as being test data set exactly once. We repeated the whole process ten times and averaged the results.

Our hypotheses on predictability are supported when the models built from our metrics provide over $70 \%$ recall or VR with less than $30 \%$ IR as described in Section IV.B.

\section{Prediction Models}

We utilized two classification techniques, linear discriminant analysis and Bayesian network, to evaluate the performance of internal metrics. The two techniques have been used in fault prediction literature $[9,18,25,26]$. 
Linear discriminant analysis is a multivariate classification technique that uses the linear function of $\mathrm{p}$ variables that achieves maximum separation among two or more groups [17]. Discriminant analysis has widespread applications, including facial recognition [21, 37]. Our other multivariate modeling technique is the Bayesian network, which is a binary classification technique based on Bayesian probability inference. In a Bayesian network, each internal metric is represented by a node in a graph, and connections between nodes exist where there is a conditional dependence observed between variables in the training set. Bayesian networks have also been used in many applications, including gene expression [32] and satellite failure monitoring systems [5].

To select the metrics for multivariate models that combine multiple metrics, we used a stepwise variable selection method, proc stepdisc, provide by $\mathrm{SAS}^{4}$ statistical analysis tool. First we selected metrics by applying stepwise selection method on discriminant analysis. We then applied the same set of metrics to Bayesian network modeling to see whether the performances of predictions are different depending on modeling techniques. Stepwise variable selection method selects one variable at a time so that the model with the selected metrics maximizes the classification between the two classes of a dependent variable [1]. If already-selected metrics do not contribute significantly to the classification based on Wilks' lambda likelihood ratio criterion [1], the variables are removed from the model. The variable selection repeats until no more metrics meet the criteria for entry $(p<.15)$ or removal $(p<.15)$.

We used SAS v9.1.3 for the discriminant analysis and Weka ${ }^{5}$ v3.6.0 for the Bayesian network modeling.

\footnotetext{
${ }^{4}$ http://www.sas.com/

${ }^{5} \mathrm{http}: / /$ www.cs.waikato.ac.nz/ml/weka/
} 


\section{CAse Study 1: Mozilla Firefox}

Our first case study has been performed on the Mozilla Firefox, a widely-used open source web browser. Mozilla Firefox had 34 releases at the time of data collection developed for four years. Each release consists of over 10,000 files and over three million lines of source code.

\section{A. Data Collection}

We collected vulnerability information from Mozilla Foundation Security Advisories (MFSAs) ${ }^{6}$. Each MFSA includes bug ids that are linked to the Bugzilla ${ }^{7}$ bug tracking system. Mozilla developers also add bug ids to the log of the CVS version control system ${ }^{8}$ when they check in the files to the CVS after the vulnerabilities have been fixed. We searched the bug ids from the CVS log to find the files that have been changed to fix vulnerabilities. The number of MFSAs for Firefox was 197 as of August, $2^{\text {nd }}, 2008$. The vulnerability fixes for the MFSAs were reported in 546 bug reports.

To collect complexity metrics, we used Understand $\mathrm{C}++{ }^{9}$, a commercial metrics collection tool. We obtained code churn and developer activity metrics from the CVS version control system.

At the time of data collection, Firefox 1.0 and Firefox 2.0.0.16 were the first and the last releases that had vulnerability reports. The gap between releases ranged from one to two months. Since the vulnerable files were too few to perform statistical analysis at each release, we combined the number of vulnerabilities for three consecutive releases and used that information to predict the vulnerabilities at the fourth releases. We collected metrics for 11 combined releases. The combined releases are denoted as R1 to R11 in this paper. We limited our analysis

\footnotetext{
${ }^{6} \mathrm{http} / / / \mathrm{www} . \mathrm{mozilla} . \mathrm{org} / \mathrm{security/announce/}$

${ }^{7}$ http://www.bugzilla.org/

${ }_{9}^{8} \mathrm{https}: / /$ developer.mozilla.org/en/Mozilla_Source_Code_Via_CVS

${ }^{9} \mathrm{http}: / / \mathrm{www}$.scitools.com
} 
to $\mathrm{C} / \mathrm{C}++$ and their header files to obtain complexity metrics. Table IV provides the project statistics for the combined 11 releases.

The big increase of the number of files in $\mathrm{R} 4$ and $\mathrm{R} 7$ indicate that there were major changes in those releases. The periods between most of the combined releases were three to four months. However, Firefox 2.0.0.8, Firefox 2.0.0.9, and Firefox 2.0.0.10 were released within a month. Lower observed fixes for R9 might be because the time gaps between releases 2.0.0.6 and 2.0.0.8 were shorter than for the other combined releases.

TABLE IV

PROJECT STATISTICS FOR MOZILlA FiREFOX

\begin{tabular}{|l|l|r|r|r|r|r|r|r|}
\hline No. & \multicolumn{1}{|c|}{ Firefox Release } & \multicolumn{1}{|c|}{$\begin{array}{c}\text { \# of } \\
\text { Files }\end{array}$} & \multicolumn{1}{|c|}{ KLOC } & $\begin{array}{c}\text { Mean } \\
\text { LOC }\end{array}$ & $\begin{array}{c}\text { Files } \\
\text { with } \\
\text { Vulns. }\end{array}$ & $\begin{array}{c}\text { Total } \\
\text { Vulns. }\end{array}$ & $\begin{array}{c}\text { Vulns. } \\
\text { per File }\end{array}$ & $\begin{array}{c}\text { \% of Files } \\
\text { with Vulns. }\end{array}$ \\
\hline R1 & $1.0 / 1.0 .1 / 1.0 .2$ & 10,320 & $3,688,444$ & 357 & 70 & 84 & 0.008 & 0.678 \\
\hline R2 & $1.0 .3 / 1.0 .4 / 1.0 .5 / 1.0 .6^{10}$ & 10,321 & $3,692,161$ & 358 & 123 & 134 & 0.013 & 1.192 \\
\hline R3 & $1.0 .7 / 1.5 / 1.5 .0 .1$ & 10,321 & $3,693,609$ & 358 & 93 & 159 & 0.015 & 0.901 \\
\hline R4 & $1.5 .0 .2 / 1.5 .0 .3 / 1.5 .0 .4$ & 10,956 & $4,012,915$ & 366 & 100 & 138 & 0.013 & 0.913 \\
\hline R5 & $1.5 .0 .5 / 1.5 .0 .6 / 1.5 .0 .7$ & 10,961 & $4,018,972$ & 367 & 109 & 153 & 0.014 & 0.994 \\
\hline R6 & $1.5 .0 .8 / 2.0 / 2.0 .0 .1$ & 10,961 & $4,022,681$ & 367 & 87 & 124 & 0.011 & 0.794 \\
\hline R7 & $2.0 .0 .2 / 2.0 .0 .3 / 2.0 .0 .4$ & 11,060 & $4,131,084$ & 374 & 114 & 162 & 0.015 & 1.031 \\
\hline R8 & $2.0 .0 .5 / 2.0 .0 .6 / 2.0 .0 .8$ & 11,060 & $4,138,242$ & 374 & 55 & 72 & 0.007 & 0.497 \\
\hline R9 & $2.0 .0 .8 / 2.0 .0 .9 / 2.0 .0 .10$ & 11,076 & $4,142,497$ & 374 & 14 & 15 & 0.001 & 0.126 \\
\hline R10 & $2.0 .0 .11 / 2.0 .0 .12 / 2.0 .0 .13$ & 11,077 & $4,142,728$ & 374 & 84 & 110 & 0.010 & 0.758 \\
\hline R11 & $2.0 .0 .14 / 2.0 .0 .15 / 2.0 .0 .16$ & 11,080 & $4,145,805$ & 374 & 27 & 46 & 0.004 & 0.244 \\
\hline
\end{tabular}

\section{B. Discriminative Power Test and Univariate Prediction Results}

All of the metrics showed statistically significant difference between vulnerable and neutral files using MWW test as shown in Table V. We also compared the mean and median of each metric measurement for two groups of files to find the association direction (positive or negative) between metrics and vulnerabilities. We performed the comparison for each release and also for the whole data across releases. The trend was similar for both of the cases. Fig. 2 shows the boxplots of comparisons of measures between the two groups for three representative metrics in

\footnotetext{
${ }^{10}$ The vulnerabilities for Firefox 1.0.5 and Firefox 1.0.6 were reported together. Therefore, we considered those two versions as one version.
} 
complexity, code churn, and developer activity metrics; CountLineCode, NumChanges, and NumDevs. The left side boxplot for each metric shows the distribution of neutral files and the right side boxplot shows the distribution of vulnerable files. The means and medians of the three metrics for the vulnerable files were higher than the ones for the neutral files as we expected.
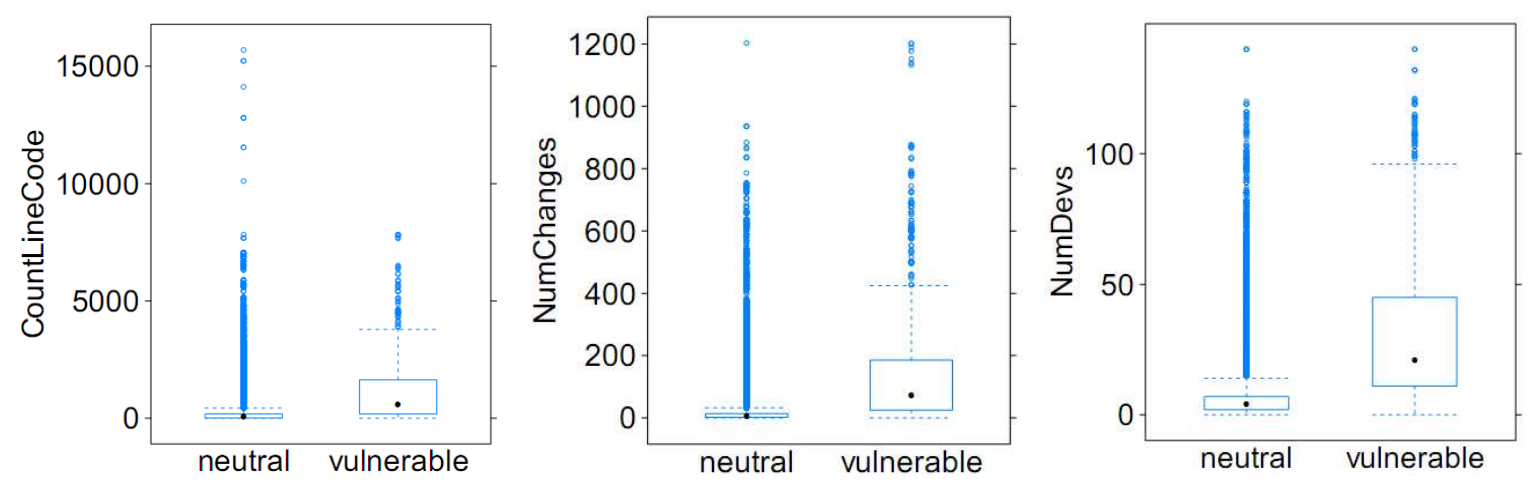

Fig. 2. Comparison of measures for vulnerable and neutral files

The results of MWW test and association directions for all the metrics are presented in Table V. The plus sign in the Association column indicates that the higher the measure, the more vulnerable the file tends to be. The minus sign indicates that the higher the measure, the less vulnerable the file tends to be. The developer degree was the only sign that did not completely agree: DNMinDegree was negatively correlated but DNAvgDegree was positively correlated. This means that files are more likely to be vulnerable if they are changed by developers who work on many other files with other developers on average, but when all of the developers are central, the file is less likely to be vulnerable. Since this is not a direct contradiction, and the other developer centrality metrics are supported, we count $\mathrm{H}_{\text {DeveloperCentrality_D hypothesis as }}$ supported. Overall, all of the hypotheses for discriminative power were supported except for DNAvgDegree.

For the predictability of individual metrics, we performed univariate discriminant analysis and validated the models using the next-release validation method. Our criterion to support the 

EDIT) $<$

hypotheses for predictability is over $70 \%$ recall or VR with less than $30 \%$ IR as described in Section IV. C. We performed the analysis on the raw data, log transformed data, and square root transformed data. Among those, log transformed data provided the best results. Table $\mathrm{V}$ shows the cross-validated prediction results using discriminant analysis on the log transformed data and results of hypotheses tests on predictability based on our criteria.

TABLE V

Results of Discriminative Power Test AND Univariate Prediction fOr MoZILla FirefoX

\begin{tabular}{|c|c|c|c|c|c|c|c|c|c|}
\hline & & & & & & rimin & t Analy & & \\
\hline Related Hypothesis & Metric & MWW & $\begin{array}{l}\text { Associ } \\
\text { ation }\end{array}$ & $\mathbf{H}_{\mathrm{D}}{ }^{*}$ & $\mathbf{R}^{*}$ & $\mathbf{P}^{*}$ & $\mathbf{I R}^{*}$ & $\mathbf{V R}^{*}$ & $\mathbf{H}_{\mathbf{P}}{ }^{*}$ \\
\hline $\mathbf{H}_{\text {Complexity }}$ & Complexity Metrics & & & $\sqrt{ }$ & & & & & $\sqrt{ }$ \\
\hline & CountLineCode & $\sqrt{ }$ & + & $\sqrt{ }$ & 79.0 & 2.1 & 28.7 & 82.9 & $\sqrt{ }$ \\
\hline & CountDeclClass & $\sqrt{ }$ & + & $\sqrt{ }$ & 52.8 & 1.1 & 32.8 & 52.5 & $\mathbf{X}$ \\
\hline & CountDeclFunction & $\sqrt{ }$ & + & $\sqrt{ }$ & 77.2 & 1.9 & 29.6 & 80.3 & $\sqrt{ }$ \\
\hline & CountLineCodeDecl & $\sqrt{ }$ & + & $\sqrt{ }$ & 79.0 & 2.2 & 26.2 & 82.7 & $\sqrt{ }$ \\
\hline $\mathrm{H}_{-}$ & CountLinePreprocessor & $\sqrt{ }$ & + & $\sqrt{ }$ & 70.1 & 2.1 & 24.1 & 75.0 & $\sqrt{ }$ \\
\hline $\mathrm{M}_{\text {UnitComplexity }}$ & SumEssential & $\sqrt{ }$ & + & $\sqrt{ }$ & 76.0 & 1.9 & 29.5 & 78.7 & $\sqrt{ }$ \\
\hline & SumCyclomaticStrict & $\sqrt{ }$ & + & $\sqrt{ }$ & 76.8 & 1.7 & 32.9 & 79.6 & $\mathbf{X}$ \\
\hline & MaxCyclomaticStrict & $\sqrt{ }$ & + & $\sqrt{ }$ & 71.0 & 1.6 & 34.5 & 74.6 & $\mathbf{X}$ \\
\hline & SumMaxNesting & $\sqrt{ }$ & + & $\sqrt{ }$ & 64.8 & 1.8 & 26.3 & 68.3 & $\mathbf{X}$ \\
\hline & MaxMaxNesting & $\sqrt{ }$ & + & $\sqrt{ }$ & 70.4 & 1.6 & 35.5 & 73.5 & $\mathbf{X}$ \\
\hline & SumFanIn & $\sqrt{ }$ & + & $\sqrt{ }$ & 71.9 & 1.7 & 30.3 & 74.4 & $\mathbf{X}$ \\
\hline & SumFanOut & $\sqrt{ }$ & + & $\sqrt{ }$ & 69.8 & 1.7 & 30.2 & 72.2 & $\mathbf{X}$ \\
\hline $\boldsymbol{\Pi}_{\text {Coupling }}$ & MaxFanIn & $\sqrt{ }$ & + & $\sqrt{ }$ & 72.3 & 1.8 & 32.6 & 74.7 & $\mathbf{X}$ \\
\hline & MaxFanOut & $\sqrt{ }$ & + & $\sqrt{ }$ & 68.2 & 1.5 & 37.1 & 71.3 & $\mathbf{X}$ \\
\hline $\mathrm{H}_{\text {Comments }}$ & CommentDensity & $\sqrt{ }$ & - & $\sqrt{ }$ & 84.4 & 1.3 & 48.9 & 86.7 & $\mathbf{X}$ \\
\hline $\mathbf{H}_{\text {CodeChurn }}$ & Code Churn Metrics & & & $\sqrt{ }$ & & & & & $\sqrt{ }$ \\
\hline $\mathrm{H}_{\text {NumChanges }}$ & NumChanges & $\sqrt{ }$ & + & $\sqrt{ }$ & 81.5 & 3.0 & 20.0 & 84.7 & $\sqrt{ }$ \\
\hline & LinesChanged & $\sqrt{ }$ & + & $\sqrt{ }$ & 81.5 & 2.4 & 24.6 & 84.6 & $\sqrt{ }$ \\
\hline $\mathrm{H}_{\text {ChurnAmount }}$ & LinesNew & $\sqrt{ }$ & + & $\sqrt{ }$ & 79.2 & 2.1 & 28.3 & 82.6 & $\sqrt{ }$ \\
\hline $\mathbf{H}_{\text {Developer }}$ & Developer Metrics & & & $\sqrt{ }$ & & & & & $\sqrt{ }$ \\
\hline & DNMinDegree & $\sqrt{ }$ & - & $\sqrt{ }$ & 57.0 & 1.2 & 36.6 & 60.5 & $\mathbf{X}$ \\
\hline & DNAvgDegree & $\sqrt{ }$ & + & $\mathbf{X}$ & 93.8 & 1.0 & 74.7 & 94.3 & $\mathbf{X}$ \\
\hline $\mathrm{H}_{-}$ & DNMaxCloseness & $\sqrt{ }$ & + & $\sqrt{ }$ & 79.8 & 1.5 & 39.0 & 82.6 & $\mathbf{X}$ \\
\hline $\mathbf{H}_{\text {DeveloperCentrality }}$ & DNAvgCloseness & $\sqrt{ }$ & + & $\sqrt{ }$ & 96.6 & 0.9 & 80.2 & 97.0 & $\mathbf{X}$ \\
\hline & DNMinBetweenness & $\sqrt{ }$ & - & $\sqrt{ }$ & 69.4 & 1.4 & 35.2 & 75.1 & $\mathbf{X}$ \\
\hline & DNAvgBetweenness & $\sqrt{ }$ & - & $\sqrt{ }$ & 96.8 & 0.9 & 82.1 & 97.6 & $\mathbf{X}$ \\
\hline$H_{-}$ & DNMaxEdgeBetweenness & $\sqrt{ }$ & + & $\sqrt{ }$ & 85.5 & 2.3 & 27.9 & 87.3 & $\sqrt{ }$ \\
\hline $\boldsymbol{\Pi}_{\text {DeveloperClustering }}$ & DNAvgEdgeBetweenness & $\sqrt{ }$ & + & $\sqrt{ }$ & 86.5 & 2.1 & 30.5 & 88.2 & $\mathbf{X}$ \\
\hline & NumDevs & $\sqrt{ }$ & + & $\sqrt{ }$ & 82.9 & 2.8 & 21.5 & 85.5 & $\sqrt{ }$ \\
\hline $\mathrm{H}_{\text {ContributionCentrality }}$ & CNCloseness & $\sqrt{ }$ & + & $\sqrt{ }$ & 81.3 & 2.7 & 22.6 & 84.4 & $\sqrt{ }$ \\
\hline & CNBetweenness & $\sqrt{ }$ & + & $\sqrt{ }$ & 64.8 & 4.8 & 9.6 & 69.7 & $\mathbf{X}$ \\
\hline
\end{tabular}

*. $\mathrm{H}_{\mathrm{D}}$ : Hypothesis test for discriminative power, R: Recall, P: Precision, IR: Inspection rate, VR: Vulnerability rate, $\mathrm{H}_{\mathrm{P}}$ : Hypothesis test for predictability 
The prior probability of a file being vulnerable or neutral for discriminant analysis was set to 0.5 in Table $\mathrm{V}$ and all the remaining analysis in this study. The bold face in the prediction results indicates that the metric satisfied the criterion for over $70 \%$ recall or VR with less than $30 \%$ IR. All of the code churn metrics supported our hypotheses for predictability. We were also able to find some sets of complexity and developer activity metrics that supported our hypotheses for predictability. While the recall and VR are over $70 \%$ for most of the metrics, the precision was very low (below 5\%) for all the metrics. Prediction with high recall and low IR is possible even with this low precision because vulnerabilities were distributed in only a small percentage of files in our data set.

Note that the metrics that provide high predictability individually do not necessarily provide high predictability together with other metrics. Conversely, the metrics that provide low predictability individually might be able to improve the overall predictability when they are used in a model together with other metrics.

\section{Multivariate Prediction Results}

We created four models using complexity, code churn, developer activity, and combination of the three categories of metrics. The independent variables were chosen using the stepwise selection method described in Section IV.D. We used only the variables with coefficients that were statistically significant at 0.05 level. We also created models with the log and square root transformed metrics for the same set of metrics chosen by stepwise selection method for discriminant analysis. Then we built models using those metrics for discriminant analysis and Bayesian network. Additionally we also built models using all the collected metrics for Bayesian network. Since the performance of Bayesian network is not affected by order-preserving data transformations, we did not apply data transformation for Bayesian network modeling. 
With discriminant analysis, log transformed data provided the best results in general. With $\log$ transformation, all of the models predicted less than $20 \%$ files as vulnerable (IR). Those predicted vulnerable files in all the models included over $70 \%$ of actual vulnerable files (recall) or over $70 \%$ of actual vulnerabilities (VR) in the system. The model with code churn metrics provided the highest recall and VR, and the combined metrics model provided the lowest IR.

With Bayesian network, the models with all the collected metrics performed better than the models with metrics selected by stepwise selection method. All of the models provided less than $20 \%$ IR. However, only the combined metrics model was able to find over $70 \%$ of actual vulnerable files (recall) with less than $20 \%$ IR. Note that recall from multivariate models has not been necessarily improved from univariate models with our data. However, IR clearly became lower with multivariate models than with univariate models. Precision was very low (less than $5 \%)$ as was in univariate models.

Table VI summarizes the cross-validated prediction results using discriminant analysis on log transformed metrics and Bayesian network on all the collected metrics. VR was not available for Bayesian network modeling with the Weka tool.

TABLE VI

Results of Multivariate PREdiction For Mozilla Firefox

\begin{tabular}{|c|c|c|c|c|c|c|c|c|}
\hline & \multicolumn{4}{|c|}{ Discriminant Analysis } & \multicolumn{3}{|c|}{ Bayesian Network } & \multirow[b]{2}{*}{$\mathbf{H}_{\mathbf{P}}$} \\
\hline & $\mathrm{R}$ & $\mathrm{P}$ & IR & VR & $\mathrm{R}$ & $\mathrm{P}$ & IR & \\
\hline Complexity & 68.9 & 3.2 & 15.4 & 72.6 & 58.0 & 3.4 & 12.5 & $\sqrt{ }$ \\
\hline Code churn & 80.5 & 3.1 & 18.6 & 84.3 & 48.2 & 9.1 & 3.8 & $\sqrt{ }$ \\
\hline Developer activity & 68.6 & 4.5 & 11.0 & 73.9 & 68.0 & 4.3 & 11.8 & $\sqrt{ }$ \\
\hline Combined & 70.8 & 4.6 & 10.9 & 76.2 & 72.1 & 4.0 & 12.9 & $\sqrt{ }$ \\
\hline
\end{tabular}

Since our metrics and models target at finding future vulnerabilities, the models should be able to provide equal or better prediction performance as time passes. Fig. 3 shows the VR across releases for the discriminant anlaysis models on the complexity, code churn, developer activity, and combined metrics. 


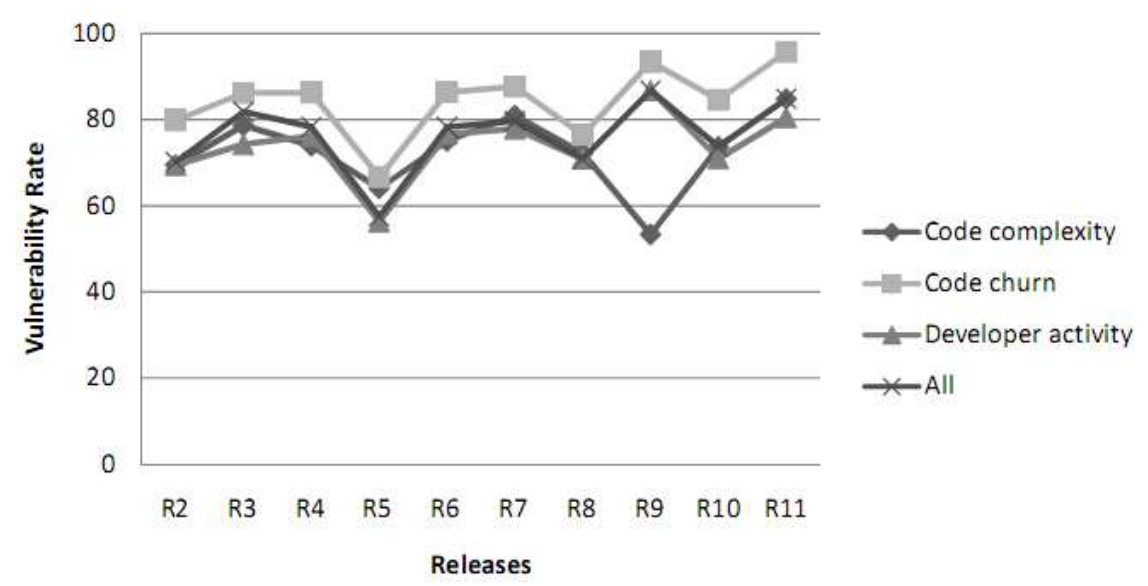

Fig. 3. Prediction results of disriminant analysis across releases

The predictive performance of the four models was slightly better for later releases than for earlier releases, which indicates that the models are trained better with the information from previous releases. The trend of recall over time was the same as that for VR. The decrease in VR of R8 might be because sudden decrease in reported vulnerabilities for R8. However, the reason of the sudden decreases in VR of R5 and R9 (complexity model) has not been identified.

\section{CASE Study 2: Red HAT ENTERPRISE LiNUX KeRNEL}

Our second case study has been performed on the Linux kernel as it was distributed in the Red Hat Enterprise Linux 4 (RHEL4) operating system. The Red Hat consists of 13,568 C and their header files with over three million lines of source code. The details of the project, data collection, and the prediction results are described in this section.

\section{A. Data Collection}

Gathering data for our external metric involved tracing through the development artifacts related to each vulnerability reported in the Linux kernel. We collected our vulnerability data from the Bugzilla database (including attached patches), the National Vulnerability Database 
$(\mathrm{NVD})^{11}$, and the Red Hat Security Advisory (RHSR) security metrics database. Since some vulnerability patches affect only certain releases, we examined each defect report manually to ensure that developers had decided that patch was, in fact, required. Since we are only interested in vulnerabilities that existed at the time of release, we did not include vulnerabilities introduced by post-release patches (a.k.a "regressions") in our data set. For vulnerabilities that did not have all of the relevant artifacts (e.g. defect reports, patches), we consulted the director of the RHSR team to correct the data. Our data set is a comprehensive list of reported, non-regression vulnerabilities from RHEL4's release in February 2005 through July 2008. We included only C and their header files. Because RHEL4 included only one C++ file, we excluded the CountDeclClass metric from our analysis.

To obtain code churn and developer activity data, we used the Linux kernel source repository ${ }^{12}$. The RHEL4 operating system is based on kernel version 2.6.9, so we used all of the version control data from kernel version 2.6.0 to 2.6.9, which was about approximately 15 months of development and maintenance. Table IX provides the project statistics.

TABLE IX

PROJECT STATISTICS FOR RED HAT LINUX

\begin{tabular}{|r|r|r|r|r|r|r|}
\hline \# of Files & LOC & Mean LOC & $\begin{array}{c}\text { Files with } \\
\text { Vulns. }\end{array}$ & Total Vulns. & $\begin{array}{c}\text { Vulns. per } \\
\text { File }\end{array}$ & $\begin{array}{c}\text { \% of Files with } \\
\text { Vulns. }\end{array}$ \\
\hline 13,568 & $3,068,453$ & 226 & 194 & 258 & 0.019 & $1.4 \%$ \\
\hline
\end{tabular}

\section{B. Discriminative Power Test and Univariate Prediction Results}

All of the metrics showed statistically significant difference between vulnerable and neutral files using MWW test except for DNMinDegree and DNMinBetweenness. All of the hypotheses for discriminative power were supported except for DNMinDegree, DNAvgDegree, DNMinBetweenness, and DNAvgBetweenness. Interestingly, DNAvgDegree was not supported

\footnotetext{
${ }^{11}$ http://nvd.nist.gov/

${ }^{12}$ http://git.kernel.org/
} 
in Mozilla either (see Section V.B). For the predictability of individual metrics, we performed univariate discriminant analysis and validated the models using ten iterations of three-fold crossvalidation. Among the metrics, SumEssential, SumMaxNesting, NumChanges, LinesNew, and NumDevs detected over $70 \%$ of vulnerable files with less than $30 \%$ IR.

Table $\mathrm{X}$ shows the results of discriminative power test and univariate prediction after crossvalidation.

TABLE X

Results of Discriminative Power TeSt AND Univariate PREDiction FOR RED HAT

\begin{tabular}{|c|c|c|c|c|c|c|c|c|c|}
\hline \multirow[b]{2}{*}{ Related Hypothesis } & \multirow[b]{2}{*}{ Metric } & \multirow[b]{2}{*}{$\begin{array}{c}\text { MW } \\
\text { W }\end{array}$} & \multirow[b]{2}{*}{$\begin{array}{c}\text { Associ } \\
\text { ation }\end{array}$} & \multirow[b]{2}{*}{$\mathbf{H}_{\mathrm{D}}$} & \multicolumn{4}{|c|}{ Discriminant Analysis } & \multirow[b]{2}{*}{$\mathbf{H}_{\mathbf{P}}$} \\
\hline & & & & & $\mathbf{R}$ & \begin{tabular}{l|l}
$\mathbf{P}$ \\
\end{tabular} & IR & VR & \\
\hline $\mathbf{H}_{\text {Complexity }}$ & Complexity Metrics & & & $\sqrt{ }$ & & & & & $\sqrt{ }$ \\
\hline \multirow{9}{*}{$\mathrm{H}_{\text {UnitComplexity }}$} & CountLineCode & $\sqrt{ }$ & + & $\sqrt{ }$ & 84.4 & 3.3 & 36.2 & 87.8 & $\mathbf{X}$ \\
\hline & CountDeclFunction & $\sqrt{ }$ & + & $\sqrt{ }$ & 79.7 & 3.5 & 32.0 & 83.3 & $\mathbf{X}$ \\
\hline & CountLineCodeDecl & $\sqrt{ }$ & + & $\sqrt{ }$ & 81.3 & 3.1 & 36.7 & 85.0 & $\mathbf{X}$ \\
\hline & CountLinePreprocessor & $\sqrt{ }$ & + & $\sqrt{ }$ & 66.7 & 2.4 & 39.3 & 69.4 & $\mathbf{X}$ \\
\hline & SumEssential & $\sqrt{ }$ & + & $\sqrt{ }$ & 82.8 & 3.9 & 29.8 & 85.9 & $\sqrt{ }$ \\
\hline & SumCyclomaticStrict & $\sqrt{ }$ & + & $\sqrt{ }$ & 82.8 & 3.6 & 32.3 & 85.8 & $\mathbf{X}$ \\
\hline & \begin{tabular}{|l|} 
MaxCyclomaticStrict \\
\end{tabular} & $\sqrt{ }$ & + & $\sqrt{ }$ & 84.1 & 3.6 & 32.8 & 86.5 & $\mathbf{X}$ \\
\hline & SumMaxNesting & $\sqrt{ }$ & + & $\sqrt{ }$ & 81.3 & 4.0 & 29.0 & 85.2 & $\sqrt{ }$ \\
\hline & MaxMaxNesting & $\sqrt{ }$ & + & $\sqrt{ }$ & 87.5 & 3.1 & 39.8 & 89.2 & $\mathbf{X}$ \\
\hline \multirow{4}{*}{$\mathrm{H}_{\text {Coupling }}$} & SumFanIn & $\sqrt{ }$ & + & $\sqrt{ }$ & 87.5 & 3.9 & 32.1 & 89.7 & $\mathbf{X}$ \\
\hline & SumFanOut & $\sqrt{ }$ & + & $\sqrt{ }$ & 84.1 & 3.6 & 33.0 & 87.0 & $\mathbf{X}$ \\
\hline & \begin{tabular}{|l|} 
MaxFanIn \\
\end{tabular} & $\sqrt{ }$ & + & $\sqrt{ }$ & 88.9 & 3.6 & 34.3 & 90.4 & $\mathbf{X}$ \\
\hline & MaxFanOut & $\sqrt{ }$ & + & $\sqrt{ }$ & 84.4 & 3.4 & 35.2 & 87.8 & $\mathbf{X}$ \\
\hline $\mathrm{H}_{\text {Comments }}$ & CommentDensity & $\sqrt{ }$ & - & $\sqrt{ }$ & 79.4 & 1.8 & 60.0 & 81.6 & $\mathbf{X}$ \\
\hline $\mathbf{H}_{\text {CodeChurn }}$ & Code Churn Metrics & & & $\sqrt{ }$ & & & & & $\sqrt{ }$ \\
\hline $\mathrm{H}_{\text {NumChanges }}$ & NumChanges & $\sqrt{ }$ & + & $\sqrt{ }$ & 81.3 & 5.4 & 21.2 & 83.0 & $\sqrt{ }$ \\
\hline \multirow{2}{*}{$\mathrm{H}_{\text {ChurnAmount }}$} & LinesChanged & $\sqrt{ }$ & + & & 81.3 & 3.2 & 36.1 & 83.7 & $\mathbf{X}$ \\
\hline & LinesNew & $\sqrt{ }$ & + & & 73.0 & 4.2 & 24.1 & 76.8 & $\sqrt{ }$ \\
\hline $\mathbf{H}_{\text {Developer }}$ & Developer Metrics & & & $\sqrt{ }$ & & & & & $\sqrt{ }$ \\
\hline \multirow{6}{*}{$\mathrm{H}_{\text {DeveloperCentrality }}$} & DNMinDegree & $\mathrm{X}$ & - & $\mathbf{X}$ & 54.7 & 1.5 & 52.7 & 52.0 & $\mathbf{X}$ \\
\hline & DNAvgDegree & $\sqrt{ }$ & + & $\mathbf{X}$ & 96.9 & 2.4 & 56.5 & 96.7 & $\mathbf{X}$ \\
\hline & DNMaxCloseness & $\sqrt{ }$ & + & $\sqrt{ }$ & 93.8 & 2.1 & 64.2 & 94.2 & $\mathbf{X}$ \\
\hline & DNAvgCloseness & $\sqrt{ }$ & + & $\sqrt{ }$ & 96.9 & 2.1 & 65.8 & 96.1 & $\mathbf{X}$ \\
\hline & DNMinBetweenness & $X$ & - & $\mathbf{X}$ & 48.4 & 1.5 & 46.7 & 51.4 & $\mathbf{X}$ \\
\hline & DNAvgBetweenness & $\sqrt{ }$ & + & $\mathbf{X}$ & 96.9 & 2.4 & 56.3 & 96.9 & $\mathbf{X}$ \\
\hline \multirow{2}{*}{$\mathrm{H}_{\text {DeveloperClustering }}$} & DNMaxEdgeBetweenness & $\sqrt{ }$ & + & $\sqrt{ }$ & 50.0 & 6.0 & 11.8 & 54.7 & $\mathbf{X}$ \\
\hline & DNAvgEdgeBetweenness & $\sqrt{ }$ & + & $\sqrt{ }$ & 50.0 & 6.0 & 11.8 & 54.7 & $\mathbf{X}$ \\
\hline \multirow{3}{*}{$\mathrm{H}_{\text {ContributionCentrality }}$} & NumDevs & $\sqrt{ }$ & + & $\sqrt{ }$ & 81.3 & 4.9 & 23.5 & 84.1 & $\sqrt{ }$ \\
\hline & CNCloseness & $\sqrt{ }$ & + & $\sqrt{ }$ & 98.4 & 1.9 & 73.3 & 97.5 & $\mathbf{X}$ \\
\hline & CNBetweenness & $\sqrt{ }$ & + & $\sqrt{ }$ & 90.6 & 3.3 & 39.2 & 92.3 & $\mathbf{X}$ \\
\hline
\end{tabular}




\section{A. Multivariate Prediction Results}

We built multivariate models using the metrics chosen by stepwise variable selection method. Table XI provides the cross-validation results of discrimininant analysis on log transformed metrics and Bayesian network on all the collected metrics for each category of metrics. With discriminant analysis, complexity, code churn, and combined metrics models provided over $70 \%$ recall or VR with less than 30\% IR. With Bayesian network modeling, complexity and combined metrics models provided over $70 \%$ recall with less than $30 \%$ IR.

TABLE XI

RESUlts OF Multivariate PREDICTION FOR RED HAT

\begin{tabular}{|c|c|c|c|c|c|c|c|c|}
\hline & \multicolumn{4}{|c|}{ Discriminant Analysis } & \multicolumn{3}{|c|}{ Bayesian Network } & \multirow[b]{2}{*}{$\mathbf{H}_{\mathbf{P}}$} \\
\hline & $\mathrm{R}$ & $\mathrm{P}$ & IR & VR & $\mathrm{R}$ & $\mathrm{P}$ & IR & \\
\hline Complexity & 79.7 & 4.5 & 25.3 & 83.9 & 70.8 & 5.0 & 20.2 & $\sqrt{ }$ \\
\hline Code churn & 78.1 & 5.4 & 20.5 & 81.3 & 21.0 & 17.2 & 1.8 & $\sqrt{ }$ \\
\hline Developer activity & 51.6 & 9.1 & 8.0 & 57.2 & 61.1 & 6.5 & 13.4 & $\mathbf{X}$ \\
\hline Combined & 68.8 & 7.5 & 13.0 & 75.3 & 73.9 & 5.5 & 19.2 & $\sqrt{ }$ \\
\hline
\end{tabular}

\section{DISCUSSION}

Most of the complexity, code churn, and developer activity metrics we used in this study were able to discriminate vulnerable files and neutral files at statistically significant level. The results from our case study support the following hypotheses across two projects.

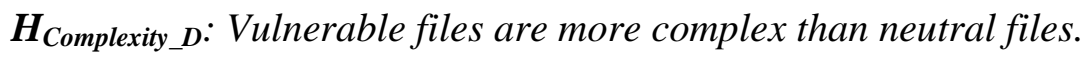

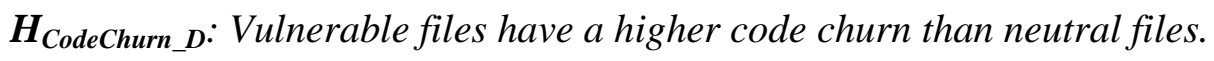

$\boldsymbol{H}_{\text {Developer_D: }}$ Vulnerable files are more likely to have been changed by poor developer activity than neutral files.

The predictive power of the metrics was different between projects and modeling techniques. Discriminant analysis performed better than Bayesian network modeling in our study. With discriminant anaylysis, seven of eight multivariate models presented in Table VI and Table XI supported our hypotheses. On the other hand, only three models supported our hypothesis with 
Bayesian network. We also performed logistic regression which is another well known classification technique. However, using logistic regression, recall for both projects was less than $50 \%$. We do not present the results of logistic regression in this paper because of the limited space. With discriminant analysis, some of the univariate models outperformed multivariate models in recall and VR. However, IRs were consistently lower for multivariate models than univariate models for both projects.

The univariate and multivariate models using complexity, code churn, and combined metrics supported our hypotheses for predictability across two projects using discriminant anlaysis and/or Bayesian network. Developer activity metrics did not support our hypotheses for predictability on Red Hat. Even in this case, however, developer activity metrics were able to predict over half of the vulnerable files at $8 \%$ IR with discriminant analysis and $13.4 \%$ IR with Bayesian network modeling. The combined metrics models provided lowest IR among the models that supported our hypotheses using discriminant analysis on both projects. Even though code churn metrics model provided high recall and low IR using discriminant analysis, the prediction performance with Bayesian network was poor. Overall there was no clear evidence whether one category of metrics is better than another because some metrics provided relatively high recall with high inspection rate while other metrics provided relatively low recall with low inspection rate. For example, code churn metrics provided higher recall than developer metrics, but also required higher IR than developer metrics. However, all of the models using the three categories of metrics and the combined metrics were able to predict vulnerabilities with over $70 \%$ recall or VR with less than $30 \%$ IR for one or both of the projects. 


\section{THREATS TO VALIDITY}

Internal validity. We performed our case studies on two open source projects whose vulnerability information is publicly available. Our analysis does not account for latent (undiscovered) vulnerabilities. Additionally, only fixed vulnerabilities are reported in detail to avoid the possible attacks from malicious users; unfixed vulnerabilities are usually not publicly available. However, considering the wide use of the subject projects and the size of community involved in the development and testing of the projects, we believe the currently reported vulnerabilities are not too limited to jeopardize our results. Also, the associations between vulnerabilities and the metrics found in this study do not necessarily indicate causation. However, we believe our results provide useful insights for organizations to take proactive or reactive actions to reduce vulnerabilities.

External validity. Our results on vulnerability prediction were not fully consistent between the two projects and the modeling techniques we used, even though complexity, code churn, and combined metrics models supported our hypotheses for both of the projects. As with all empirical studies, our results were limited to the metrics we collected and the modeling techniques we used in this study. To generalize our observations from this study to other projects in various domain, size, and operational and usage context, further studies should be performed. We believe our results can be used to compare with the results from other projects and to increase the understanding on the relationship between software vulnerabilities and the metrics investigated in this study.

\section{SUMMARY}

Our study explored the relationship between complexity, code churn, and developer activity metrics with vulnerabilities. Complexity and developer activity metrics are actionable metrics 
that organizations can take actions to change the measures of metrics by redesigning code or reorganizing a development team when necessary. In our case study on Mozilla Firefox and Red Hat Enterprise Linux kernel, those actionable metrics showed discriminative power between vulnerable and neutral files. Therefore, organizations can use complexity and developer activity metrics to proactively improve software security. For example, while some code cannot avoid complexity, organizations can use the average values of complexity metrics for vulnerable files and neutral files as a criterion to redesign unnecessarily complex code. Central developers tend to produce less vulnerabilities than developers who work alone. Therefore, appropriate corrective action in a development team such as pair programming or careful distribution of central developers based on the measures of developer activity metrics is encouraged.

When metrics can predict vulnerabilities, those predictive metrics can be used to prioritize inspection and testing effort. In our case study, all three categories of metrics had predictability at one or both projects by providing over $70 \%$ recall or vulnerability rate with less than $30 \%$ inspection rate. Code churn metrics provided the best recall for Mozilla Firefox among the models using the three categories of metrics and combined metrics using discriminant analysis. Complexity metrics provided the best recall for Red Hat using discriminant analysis. However, there was no clear evidence whether one category of metrics was better than another. Whether the prediction results are good or not can be determined by practitioners at organizations. However, finding $84.3 \%$ of vulnerabilities by inspecting only $18.6 \%$ of total files (in the case of code churn metrics model with discriminant analysis on Mozilla Firefox) might be a promising result to appropriately allocate inspection efforts. Our results are promising. However, additional studies of these and other metrics could lead to further improvement. 


\section{ACKNOWLEDGMENT}

We thank the Mozilla team who clarified the procedure for version control and vulnerability fixes. We thank Mark Cox, the director of the RHSR team for verifying our Red Hat data. We also thank the NCSU Software Engineering Realsearch group for their careful review of and helpful suggestions for the paper.

\section{REFERENCES}

[1] "SAS/STAT 9.1 User's Guide", SAS Institute Inc., 2004.

[2] "IEEE Standard for a Software Quality Metrics Methodology", IEEE Std 1061-1998 (R2004), IEEE Computer Society, 2005.

[3] Alhazmi, O.H., Malaiya, Y.K., and Ray, I., "Measuring, Analyzing and Predicting Security Vulnerabilities in Software Systems", Computers \& Security, 26(3), 2007, pp. 219-228.

[4] Basili, V.R., Briand, L.C., and Melo, W.L., "A Validation of Object-Oriented Design Metrics as Quality Indicators", IEEE Trans. Software Eng., 22(10), 1996, pp. $751-761$.

[5] Bottone, S., Lee, D., O'Sullivan, M., and Spivack, M., "Failure Prediction and Diagnosis for Satellite Monitoring Systems using Bayesian Networks", Proc. Military Communications Conference, 2008 (MILCOM'08), San Diego, CA, USA, 16-19 Nov., 2008, pp. 1-7.

[6] Brandes, U., and Erlebach, T., Network Analysis: Methodological Foundations, Lecture Notes in Computer Science, Springer, 2005.

[7] Briand, L.C., Wüst, J., Daly, J.W., and Porter, D.V., "Exploring the Relationships Between Design Measures and Software Quality in Object-Oriented Systems", Journal of Systems and Software, 51(3), 2000, pp. 245-273.

[8] Cashell, B., Jackson, W.D., Jickling, M., and Web, B., "CRS Report for Congress: The Economic Impact of Cyber-Attacks", Congressional Research Service, April 1, 2004.

[9] Fenton, N., Neil, M., Marsh, W., Hearty, P., Radlinski, L., and Krause, P., "Project Data Incorporating Qualitative Facts for Improved Software Defect Prediction ", Proc. the Third International Workshop on Predictor Models in Software Engineering (PROMISE'07), Washington, DC, USA, 20-26 May, 2007

[10] Geer, D.E., "A Witness Testimony in the Hearing, Wednesday 25 April 07, entitled Addressing the Nation's Cybersecurity Challenges: Reducing Vulnerabilities Requires Strategic Investment and Immediate Action", submitted to the Subcommittee on Emerging Threats, Cybersecurity, and Science and Technology, 2007

[11] Gegick, M., Rotella, P., and Williams, L., "Toward Non-Security Failures as a Predictor of Security Faults and Failures", Proc. International Symposium on Engineering Secure Software and Systems (ESSoS), Leuven, Belgium, February 04-06, 2009, pp. 135-149.

[12] Gegick, M., Williams, L., Osborne, J., and Vouk, M., "Prioritizing Software Security Fortification through Code-Level Metrics", Proc. 4th ACM workshop on Quality of protection, Alexandria, Virginia, USA, Oct. 27, 2008, pp. 31-38.

[13] Girvan, M., and Newman, M.E.J., "Community Structure in Social and Biological Networks", the National Academy of Sciences, 99(12), 2001, pp. 7821-7826.

[14] Gonzales-Barahona, J.M., Lopez-Fernandez, L., and Robles, G., "Applying Social Network Analysis to the Information in CVS Repositories", Proc. International Workshop on Mining Software Repositories (MSR'04), Edinburgh, Scotland, UK, 25 May, 2004

[15] Hudepohl, J.P., Jones, W., and Lague, B., "EMERALD: A Case Study in Enhancing Software Reliability", Proc. International Symposium on Software Reliability Engineering (ISSRE '97), Albuquerque, NM, 2-5 Nov, 1997, pp. 85-91.

[16] "ISO/IEC TR 9126-2 Software engineering — Product quality Part 2:External metrics", ISO/IEC TR 9126-2:2003(E), 07-01, 2003.

[17] Johnson, R.A., and Wichrn, D.W., Applied Multivariate Statistical Analysis, Prentice Hall, Upper Saddle River, NJ, USA, 2002.

[18] Khoshgoftaar, T.M., Allen, E.B., Kalaichelvan, K.S., and Goel, N., "Early Quality Prediction: A Case Study in Telecommunications", IEEE Software, 13, (1), Jan., 1996, pp. 65 - 71.

[19] Krsul, I.V., "Software Vulnerability Analysis", PhD dissertation, Purdue University, 1998.

[20] Mann, H.B., and Whitney, D.R., "On a Test of Whether One of Two Random Variables is Stochastically Larger than the Other", the Annals of Mathematical Statistics, 18, 1947, pp. 50-60.

[21] Martinez, A.M., and Kak, A.C., "PCA versus LDA", IEEE Trans. Pattern Analysis and Machine Intelligence, 23(2), 2001, pp. $228-233$.

[22] McCabe, T.J., "A Complexity Measure", IEEE Trans. Software Eng., 2(4), 1976, pp. 308-320.

[23] McGraw, G., Software Security: Building Security In, Addison-Wesley, Boston, NY, 2006.

[24] Meneely, A., Williams, L., Snipes, W., and Osborne, J., "Predicting Failures with Developer Networks and Social Network Analysis", Proc. 16th ACM SIGSOFT International Symposium on Foundations of Software Engineering, Atlanta, Georgia, Nov. 9-14, 2008, pp. 13-23.

[25] Menzies, T., Greenwald, J., and Frank, A., "Data Mining Static Code Attributes to Learn Defect Predictors", IEEE Trans. Software Eng., 33(1), 2007, pp. 2 - 13.

[26] Nagappan, N., and Ball, T., "Use of Relative Code Churn Measures to Predict System Defect Density", Proc. the 27th International Conference on Software Engineering (ICSE '05), St. Louis, MO, USA, May 15-21, 2005, pp. 284 - 292.

[27] Nagappan, N., and Ball, T., "Using Software Dependencies and Churn Metrics to Predict Field Failures: An Empirical Case Study", Proc. First International Symposium on Empirical Software Engineering and Measurement, Madrid, Spain, 20-21 Sept., 2007, pp. 364 - 373.

[28] Nagappan, N., Ball, T., and Murphy, B., "Using Historical In-Process and Product Metrics for Early Estimation of Software Failures", Proc. the 17th International Symposium on Software Reliability Engineering, Raleigh, NC, U.S.A., November 7-10, 2006, pp. 62-74.

[29] Nagappan, N., Ball, T., and Zeller, A., "Mining Metrics to Predict Component Failures", Proc. the 28th International Conference on Software Engineering (ICSE'06), Shanghai, China, May 20-28, 2006, pp. 452-461. 
[30] Nagappan, N., Murphy, B., and Basili, V.R., "The Influence of Organizational Structure on Software Quality", Proc. International Conference on Software Engineering, Leipzig, Germany, 10 - 18 May, 2008, pp. 521-530.

[31] Neuhaus, S., Zimmermann, T., and Zeller, A., "Predicting Vulnerable Software Components", Proc. the 14th ACM Conference on Computer and Communications Security (CCS'07), Alexandria, Virginia, USA, October 29-November 2, 2007, pp. 529 - 540.

[32] Numata, K., Imoto, S., and Miyano, S., "A Structure Learning Algorithm for Inference of Gene Networks from Microarray Gene Expression Data Using Bayesian Networks", Proc. Bioinformatics and Bioengineering, Boston, MA, USA., 14-17 Oct., 2007, pp. 12801284.

[33] Ostrand, T.J., Weyuker, E.J., and Bell, R.M., "Predicting the Location and Number of Faults in Large Software Systems", IEEE Trans. Software Eng., 31(4), 2005, pp. 340 - 355.

[34] Pinzger, M., Nagappan, N., and Murphy, B., "Can Developer-Module Networks Predict Failures?", Proc. International Symposium on Foundations in Software Engineering, Atlanta, GA, 9-14 Nov., 2008, pp. 2-12.

[35] Schneidewind, N.F., "Methodology for Validating Software Metrics", IEEE Trans. Software Eng., 18, 1992, pp. 410-422.

[36] Shin, Y., and Williams, L., "Is Complexity Really the Enemy of Software Security?", Proc. the 4th ACM Workshop on Quality of Protection, Alexandria, Virginia, USA, Oct. 27, 2008, pp. 47-50.

[37] Tae-Kyun, K., and Kittler, J., "Locally Linear Discriminant Analysis for Multimodally Distributed Classes for Face Recognition with a Single Model Image", Pattern Analysis and Machine Intelligence, IEEE Transactions on, 27(3), 2005, pp. 318-327.

[38] Tenny, T., "Program Readability: Procedures Versus Comments", IEEE Trans. Software Eng., 14(9), 1988, pp. 1271-1279.

[39] Watson, A.H., and McCabe, T.J., "Structured Testing: A Testing Methodology Using the Cyclomatic Complexity Metric", NIST Special Publication 500-235, September, 1996.

[40] Weissman, L., "Psychological Complexity of Computer Programs: an Experimental Methodology", ACM SIGPLAN Notices, 9, (6), 1974, pp. 25 - 36.

[41] Weyuker, E.J., Ostrand, T.J., and Bell, R.M., "Using Developer Information as a Factor for Fault Prediction ", Proc. International Workshop on Predictor Models in Software Engineering (PROMISE '07), Minneapolis, MN, 20 May, 2007, pp. 8.

Yonghee Shin is a PhD student in the Computer Science Department at North Carolina State University (NCSU) under the supervision of Dr. Laurie Williams. Her research interests are software testing and software quality assurance specialized in software security. She received her MS degree from Texas A\&M University in 2003 and BS degree from Sookmyung Women's University in 1992. She worked for Daewoo Telecommunications and Samsung SDS for eight years in Seoul, Korea before returning to academia. She is a student member of the IEEE.

Andrew Meneely is a Ph.D. student in the Computer Science Department at NCSU under the supervision of Dr. Laurie Williams. His research interests include empirical software engineering, software security, and developer collaboration. He received his MS degree from NCSU in 2008 and received a dual BA in Computer Science and Mathematics from Calvin College in 2006. He is a student member of IEEE.

Laurie Williams is an Associate Professor in the Computer Science Department at NCSU. Her research focuses on agile software development practices and processes; software reliability, software testing and analysis; software security; open source software development; and broadening participation and increasing retention in computer science. Laurie is the Director of the North Carolina State University Laboratory for Collaborative System Development and the Center for Open Software Engineering, and an area representative for the Secure Open Systems Initiative. Laurie is the technical co-director of the Center for Advanced Computing and Communication (CACC). Laurie received her Ph.D. in Computer Science from the University of Utah, her MBA from Duke University Fuqua School of Business, and her BS in Industrial Engineering from Lehigh University. She worked for IBM Corporation for nine years in Raleigh, NC and Research Triangle Park, NC before returning to academia. She is a member of the IEEE.

Jason Osborne is an Associate Professor in the Department of Statistics at NCSU. Most of his effort goes towards providing internal statistics consulting to the university and his research is driven by problems that arise from that work. Examples include estimation of linear Boolean models for particle flow, estimation of population size in software reliability and heterogeneity of variance models for gene expression experiments. Jason received a PhD in Statistics from Northwestern University and a BS in Mathematics from the University of California at Santa Barbara. 